\title{
Numerical simulation and analysis of heat dissipation characteristics of battery box of electric vehicle based on coupling model
}

\author{
WEI Jia-tong ${ }^{1}$, KONG Chun-hua ${ }^{2}, X U$ Yan $^{3}$ \\ \{ouyangmeixue100@163.com ${ }^{1}, 13596157766 @ 163 . c^{2}$, xuyan464803398@163.com³ \\ (1.Department of Automotive Engineering,Jilin Communications Polytechnic,Changchun 130012,China; \\ 2.Department of Automotive Engineering,Jilin Communications Polytechnic,Changchun 130012,China; \\ 3.Department of Automotive Engineering,Jilin Communications Polytechnic,Changchun 130012,China)
} Abstract: In order to reduce the heat loss of battery in the process of electric vehicle moving, a numerical simulation analysis method based on coupling model is proposed. According to the actual consumption demand of the heat dissipation structure of the battery box, the center difference value level of the heat dissipation coefficient is calculated, and then the heat dissipation coefficient of the battery box of the electric vehicle is processed according to the separation principle of the boundary heat dissipation authority. On this basis, a standardized simulation grid is established by combining the characteristic coupling numerical value, and the comprehensive simulation processing of the thermal characteristic numerical value is realized by calculating the angle deflection of the thermal domain of the battery, and the successful application of the numerical simulation analysis method of the thermal characteristic of the battery box of the electric vehicle based on the coupling model is completed. The results show that, compared with the conventional numerical processing, the DSI and PSI coefficients of the new numerical simulation method show a significant downward trend, and the heat loss of the battery in the process of electric vehicle moving is effectively controlled.

Keywords: coupling model; electric vehicle; heat dissipation characteristics; numerical simulation; consumption demand; center difference value; simulation grid; heat dissipation domain deflection;

\section{Introduction}

In electronics, coupling refers to the transfer of energy from one circuit part to another. For example, through electrical conductivity coupling, energy is transmitted from a voltage source to the load. The AC (Alternating Current) part and DC (Direct current) part of the circuit can be coupled by using the properties of the capacitor that allows the AC part to pass through and block the DC part. The transformer can also act as the coupling medium, and the appropriate impedance matching can be achieved by configuring the appropriate impedance at both ends. In practical engineering, temperature field, gravitational field and humidity field are 
all physical fields, and many of the problems we need to solve are the superposition of these physical fields, because these physical fields interact with each other. For example, the temperature has an effect on the stress distribution. The problem of superposition of multiple physical fields is called multi field coupling problem, which is also a kind of coupling. Simply put, in software engineering, the coupling degree between objects is the dependency between objects [1]. The main problem guiding the use and maintenance of objects is the multiple dependencies between objects. The higher the coupling between objects, the higher the maintenance cost. Therefore, the design of objects should minimize the coupling between classes and components.

The heat dissipation technology is derived from the traditional heat dissipation technology, which is based on conduction and convection. In the field of security, the heat dissipation technology is applied to the heat dissipation of security monitoring equipment to improve the working performance and stability of the equipment. The heat dissipated in this way depends on the temperature difference between the body temperature and the contact object, the contact area, and the thermal conductivity of the object in contact with the body to dissipate heat. Combined with the convective heat dissipation technology, the heat from the body is exchanged through the gas flow. The amount of heat dissipated by convection depends on the temperature difference between the fuselage and the surrounding environment and the effective cooling area of the fuselage, which is greatly affected by the wind speed [2]. The greater the wind speed, the more heat dissipation; on the contrary, the smaller the wind speed, the less heat dissipation. In general, the contact surface between the electronic components and the heat sink should be coated with a layer of thermal conductive silicone grease, so that the heat from the components can be more effectively transmitted to the heat sink, and then distributed to the surrounding air through the heat sink. In the conventional simulation scheme, the part close to the front cover of the battery box and the car is changed to copper block, which has the characteristics of fast heat absorption and strong heat conduction ability, so as to quickly a large amount of heat energy generated by the operation of the module is brought to the copper block with nickel plating on the surface, and the copper block and the front cover aluminum block are closely combined to dissipate heat, so that a large amount of heat energy can quickly spread to the aluminum alloy body, and then be taken away by the flow of gas. Combined with the heat dissipation technology of the battery, an open space is provided for the heat loss of the battery module, so as to ensure that the equipment has enough space to realize air convection and effectively The battery box of electric vehicle is ensured to work in the daily environment. The heat dissipation of battery box is a hot research issue in the field of heat dissipation technology. Under the condition of high-speed driving of electric vehicle, a 
new numerical simulation analysis method of heat dissipation characteristics of battery box is designed based on the theory of domain angle deflection. The practical application value of this method is highlighted by the way of comparative experiment.

\section{Heat dissipation coefficient treatment of battery box of electric vehicle}

The treatment of the heat dissipation coefficient of the battery box of the electric vehicle consists of three parts: the analysis of the heat dissipation structure of the battery box, the calculation of the center difference of the heat dissipation coefficient and the separation of the boundary heat dissipation authority. The specific operation method is as follows.

\subsection{Thermal structure analysis of battery box}

The working cycle of the electric vehicle engine is carried out under high temperature, and the temperature of the mixture entering the cylinder can reach over $2000{ }^{\circ} \mathrm{C}$. At this time, the piston, cylinder block, cylinder head, valve and other parts of the engine are in contact with the high-temperature combustible mixture and are heated intensively. If the engine is not cooled effectively, its mechanical strength will become worse. At the same time, the inflation coefficient of the cooling structure will decrease, resulting in the imbalance of air-fuel ratio and abnormal combustion of the engine. However, the high temperature in the battery box will cause the mixture to burn early (burn in advance), resulting in the knock phenomenon that seriously damages the electric vehicle [3]. Too high temperature will also cause the lubricating oil to burn and deteriorate. At high temperature, it will reduce the gap in the heat dissipation structure, damage the protection of the oil film, reduce the lubricating capacity, and even cause adhesion wear and seizure (cylinder pulling) fault.

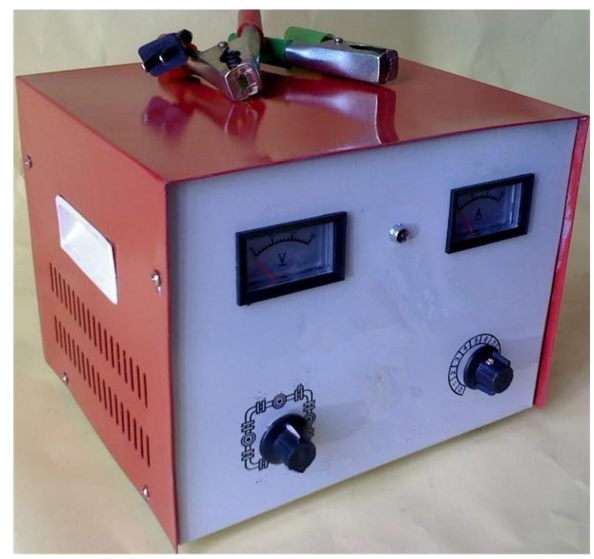

Fig. 1 Heat dissipation structure of battery box

\subsection{Calculation of center difference value of heat dissipation coefficient}

The central difference value is the necessary performance condition of the heat 
dissipation structure of the battery box, because the heat dissipation coefficient of the battery box of the electric vehicle not only retains the Taylor series information in the first-order upwind, but also retains the second term of the Taylor series, so the precision of the central difference value is higher than that of the first-order upwind format. The first-order upwind scheme considers that the value on the upstream cell control point is equal to the value of the local cell boundary point. The second-order upwind scheme considers that the value of the boundary point of the local element is equal to the sum of the value and increment of the control point of the upstream element[4-5]. The second-order upwind scheme is commonly used to solve vehicle flow field. In order to obtain the second-order accuracy of the flow field variables on the boundary point, the control point of the local grid element can also be taken as the base point. Similarly, the flow field variables are expanded by taylor series and the first two terms are retained. Generally, the value of the second-order upwind scheme is different from that of the boundary point. The value of the flow field variable calculated by the central difference scheme at the boundary point is the arithmetic mean of the two. Let $q_{0}$ represent the two lower limit accumulation conditions of the heat dissipation coefficient of the battery box, $q_{1}$ represent the two upper limit accumulation conditions of the heat dissipation coefficient of the battery box, and $\lambda$ represent the control increment of the battery unit. The central difference value of the heat dissipation coefficient can be expressed as:

$$
\delta=\int_{q_{0}}^{q_{1}} \sqrt{\frac{y}{\lambda-1}}\left(\frac{j}{f}\right)^{2} \cdot|\bar{h}| d \bar{h}
$$

Among them, $y$ represents the boundary value of the local cell, $j$ represents the first-order upwind scheme, $f$ represents the second-order upwind scheme, and $\bar{h}$ represents the characterization index of flow field variables in Taylor series.

\subsection{Separation and solution of boundary heat dissipation authority}

As the name implies, the separation and solution of boundary heat dissipation authority is to deal with different heat dissipation structures of battery box of electric vehicle. Because of the nonlinear characteristics of the control equation, the convergence solution can be obtained only after several iterations. Figure 2 is the solution flow chart. The process is as follows:

(1) Update the heat dissipation characteristic value of the battery box of the electric vehicle. In the first calculation, the initial value of the variable is assigned by initialization. In the later calculation process, each iteration gets a new solution. 
(2) A new characteristic field strength is obtained by solving the momentum equation from the current mass and pressure flux values of the cell box.

(3) Because the numerical solution of the characteristic field strength can't meet the continuous equation completely, it is necessary to solve the Poisson equation derived from the continuous equation again, and solve the equation to modify the heat flux, heat pressure and driving speed field, so as to meet the continuous equation.

(4) Through the solutions obtained above, the equations of components, energy and turbulence are solved.

(5) If interphase interference is considered in multiphase flow calculation, the source term solution in the continuous phase equation needs to be solved again.

(6) Check whether the convergence condition is met. If so, the calculation is terminated. On the contrary, continue to iterate.

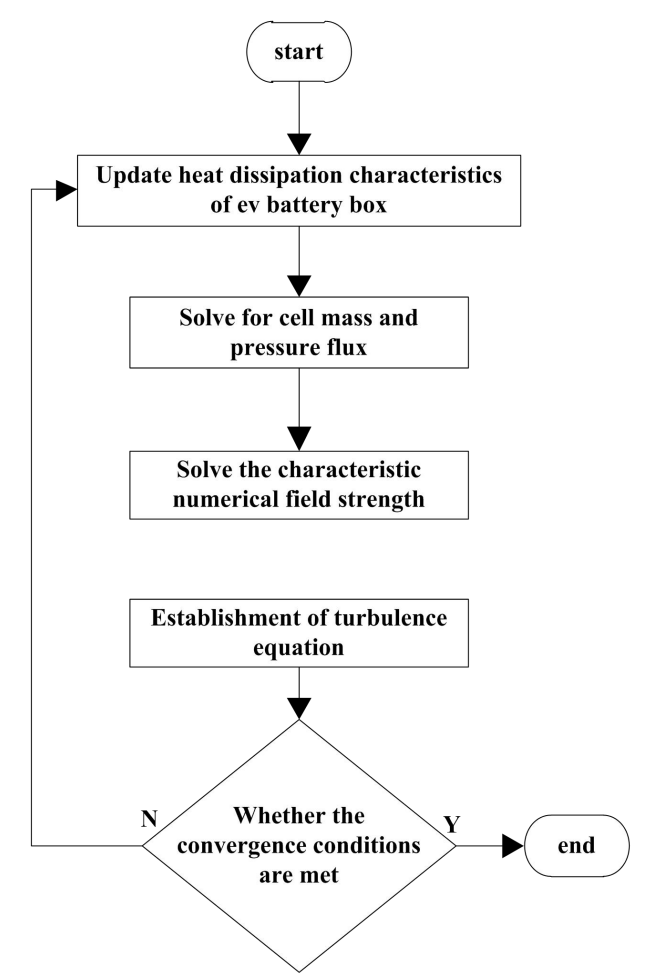

Fig. 2 Flow chart of boundary heat dissipation authority separation

\section{Numerical simulation method of heat dissipation characteristics based on coupled model}

Under the support of the treatment condition of the heat dissipation coefficient of the electric vehicle battery box, according to the process of building the numerical simulation grid 
of the characteristics coupling, calculating the angle deflection of the magnetic domain of the heat dissipation of the battery, and comprehensively simulating the numerical value of the heat dissipation characteristics, the design of the numerical simulation analysis method of the heat dissipation characteristics of the electric vehicle battery box based on the coupling model is completed.

\subsection{Grid construction of characteristic coupled numerical simulation}

In the characteristic numerical simulation grid, the numerical simulation of the heat dissipation characteristics of the electric vehicle battery needs to discretize the spatial domain of the calculation range to solve the discretized equations, and the discretization process is based on the grid. In the process of calculation, all kinds of physical information of flow field are stored in the grid nodes. Therefore, the grid has a direct impact on the accuracy of the calculation results. In reality, when the car is running in the air, the surrounding air tends to be infinite, and the simulation can not include all the space, and in the distance from the car, the air disturbance by the car is very small [6]. In the characteristic numerical simulation grid, the heat sink is the same as the battery electron, and can only transfer from one direction to another. This trend will never be reversed throughout the analysis. In the application of the characteristic devices of the electric vehicle battery radiator, the external detection coil is generally used to realize the sensing of magnetic signal, and the analysis of the influence of the device structure, effective magnetic field distribution, material permeability and other factors on the sensing results is simple. In the process of numerical analysis of self sensing characteristics, there is no external detection coil, so it is necessary to extract the magnetic sensing signal from the excitation signal of the excitation coil. How to reduce the interference of the excitation signal to the magnetic sensing signal is the first choice; and the sensed battery signal is the change of the magnetic field in three parts, including giant magnetostrictive material, nylon framework and excitation coil, in addition to optimized implementation. In the optimized structure, besides the uniformity of the magnetic field inside the device, the influence of the uneven distribution of the magnetic field inside the giant magnetostrictive material and the different values of the permeability of the material on the magnetic sensing results should also be considered [7]. Therefore, it is necessary to establish a precise mathematical sensing relationship including actuator structure and magnetic field optimization, effective magnetic field calculation in the actuator and the influence of permeability of giant magnetostrictive materials. 


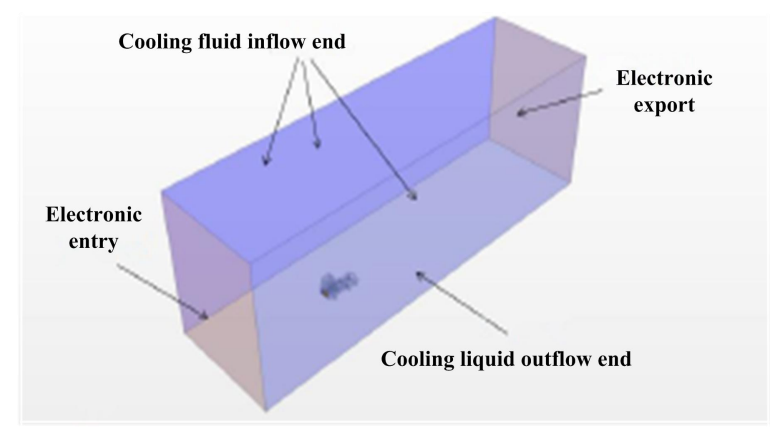

Fig. 3 Characteristic numerical simulation grid

\subsection{Calculation of domain angle deflection for cell heat dissipation}

In the process of domain deflection of battery cooling materials, the effective path of domain deflection is relatively complex, which mainly depends on the very large anisotropic properties of the materials. In addition to the magnetocrystalline anisotropic energy of the electric vehicle itself, the application of stress field will also produce a stress anisotropic property in the materials, which will make the deflection process of the domain under the load More complex [8]. Under the action of stress coupling model and heat dissipation load, the change of free energy in each part of the material will make the domain angle deflect. In the study of the domain deflection model of giant magnetostrictive materials, table 1 lists a group of typical constraints between the heat dissipation coefficient and the domain angle deflection.

Table 1 Statistical table of coefficient restriction relationship

\begin{tabular}{ccc}
\hline $\begin{array}{c}\text { Heat dissipation coefficient } \\
\text { of battery }\end{array}$ & $\begin{array}{c}\text { Domain angle } \\
\text { deflection }\end{array}$ & Restrictive relationship \\
\hline$\alpha_{1}$ & Inhibition \\
$\alpha_{2}$ & Promote \\
$\beta_{1}$ & Inhibition \\
$\beta_{2}$ & Promote \\
$p$ & & Promote \\
$w$ & Inhibition \\
\hline
\end{tabular}

In the above table, the heat dissipation coefficient of the battery from top to bottom successively represents the lower limit characteristic value, upper limit characteristic value, minimum coupling error, maximum coupling error, heat dissipation coefficient of the battery 
box and the traveling parameters of the electric vehicle. With the support of the above physical quantities, the joint formula (1) can express the calculation result of the angle deflection of the magnetic domain of the battery heat dissipation as follows:

$$
\delta=\frac{\left|\int_{\alpha_{1}}^{\alpha_{2}}\left(p-t t^{\prime}\right)^{2} d t\right|}{\sqrt{\int_{\beta_{1}}^{\beta_{2}} w \cdot\left\|l+l^{\prime}\right\| d l}}
$$

Among them, $t$ represents the thermal load condition of the battery box of the electric vehicle, $t^{\prime}$ represents the numerical supplementary authority index of $t, l$ represents the primary deflection coefficient of the thermal domain angle, and $l^{\prime}$ represents the numerical supplementary condition of $l$.

\subsection{Comprehensive simulation of heat dissipation characteristics}

From the point of view of the structural behavior of deflection from the perspective of the thermal domain of the battery, the excitation magnetic field provided to the giant magnetostrictive material is controlled by adjusting the magnitude of the excitation current in the battery box, so as to drive the electric vehicle to generate the mechanical stress, in which the mechanical structure will bear the application of the preload stress and the output of the mechanical stress, thus affecting the actual application effect of the coupling model. The actuator of battery box just uses the magnetostrictive mechanism of giant magnetostrictive material itself to realize the conversion of magnetic energy to mechanical energy, and outputs the mechanical stress and displacement through the car top bar [9]. Considering the working characteristics of giant magnetostrictive materials, the actuator of the battery box will sometimes be equipped with a heat dissipation magnetic field, which is mainly provided by characteristic bias coil or coupling device to eliminate the "dual frequency characteristics" of the electric vehicle itself; the automobile top bar is mainly used to provide a prepressing stress required for the work of materials with heat dissipation characteristics, so as to make the materials work in a better strain area, so as to improve the energy conversion efficiency of the battery box actuator; the cooling mechanism is mainly used for the temperature rise caused by the internal loss of the battery box actuator, so as to reduce the impact of the change of heat dissipation temperature on the performance of the actuator, including forced water cooling and phase change Temperature control and semiconductor refrigeration. The specific processing flow is shown in Figure 4 


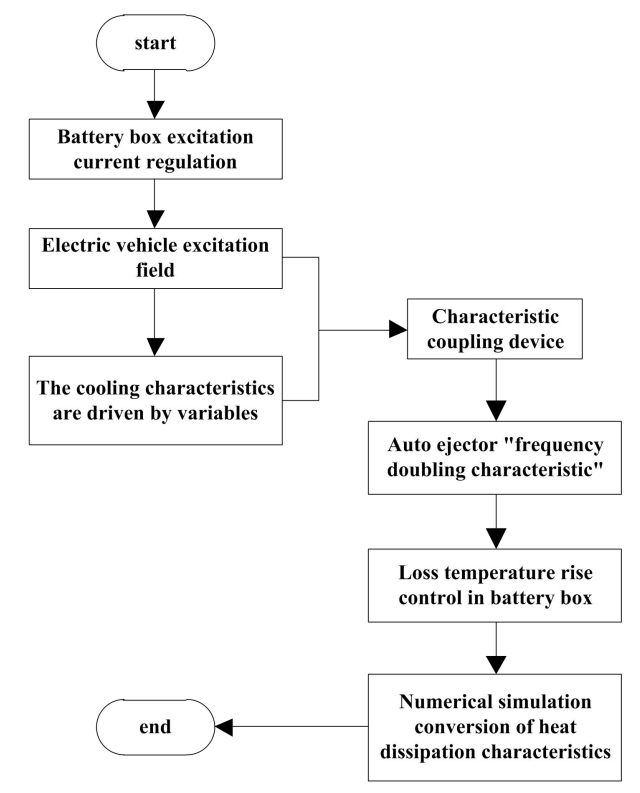

Fig. 4 Flow chart of numerical integrated simulation processing of heat dissipation characteristics

The self sensing application of the battery box actuator is an organic collection of execution and perception. The driving and characteristic sensing functions of the electric vehicle are displayed in the material in real time. The self sensing application is completed through the coupling model analysis and characteristic numerical simulation of the material. Therefore, the coupling of heat dissipation materials and the identification and extraction of electromagnetic parameters will be the key to realize the application of material self sensing [10]. Among them, the coupling model of heat dissipation material and the application model of battery box actuator are the premise of realizing self sensing application. In terms of material coupling model, the internal coupling relationship between input heat dissipation parameters and output characteristic values is established, and the change of state parameters under different loads is the basis of defining the coupling relationship model, which is applicable to the constitutive relationship establishment of self sensing application It is the foundation of actuator application model research; In the aspect of the application model of battery box actuator, the mathematical establishment of heat dissipation characteristics and the perception of coupling parameters will be the key to the application of actuator perception. The optimal design of heat dissipation characteristics and the mathematical calculation of accurate indicators directly affect the perception accuracy of the model.

Let $f$ represent the coupling coefficient of the heat dissipation characteristic material, 
and $\tilde{k}$ represent the disposal conditions of the application model of the battery box actuator. The combined formula (2) can express the comprehensive simulation results of the heat dissipation characteristic values as follows:

$$
c=\sqrt{\frac{\sum_{n=1}^{b}\left(f+f^{\prime}\right)^{2} \dot{g}}{3 \tilde{k} \beta / \lambda \cdot|\bar{x}|}} \text { (3) }
$$

Among them, $n$ represents the lower deflection coefficient of the characteristic heat dissipation value of the electric vehicle battery heat sink, $b$ represents the upper deflection coefficient of the characteristic heat dissipation value of the electric vehicle battery heat sink,

$f^{\prime}$ represents the opposite characteristic number of the coupling coefficient, $\dot{g}$ represents the comprehensive simulation authority, $\beta$ represents the simulation cycle of implementing the heat dissipation characteristic value, $\lambda$ represents the heat dissipation index vector of the electric vehicle battery box, $\bar{x}$ represents the heat dissipation characteristic The average simulation condition of the numerical value.

\section{Simulation analysis and verification}

In order to verify the effectiveness of the numerical simulation analysis method based on the coupling model, the following comparative experiments are designed. Two electric vehicle battery heat sinks with the same model and output effect are selected as the experimental objects. The heat loss behavior of the heat sink in the experimental group is monitored by the characteristic numerical simulation analysis method, and the heat loss behavior of the control group is monitored by the conventional numerical processing method. The specific changes of the relevant index parameters are recorded in the established experimental time.

\subsection{Establishment of simulation inspection environment}

By means of manual intervention, the numerical monitoring methods applied in the experimental group and the control group were changed. With the aid of transmission wire, the recorded index parameters were fed back to the core simulation host. 


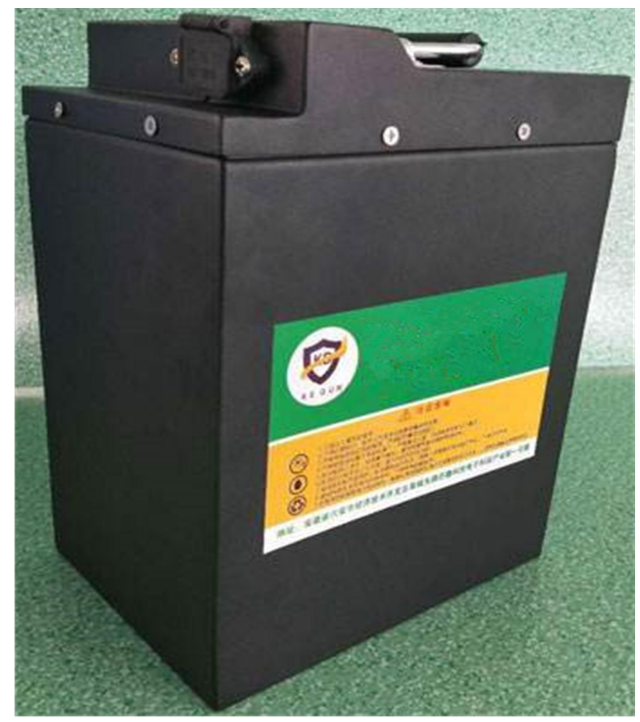

Fig. 5 Electric vehicle battery cooling box

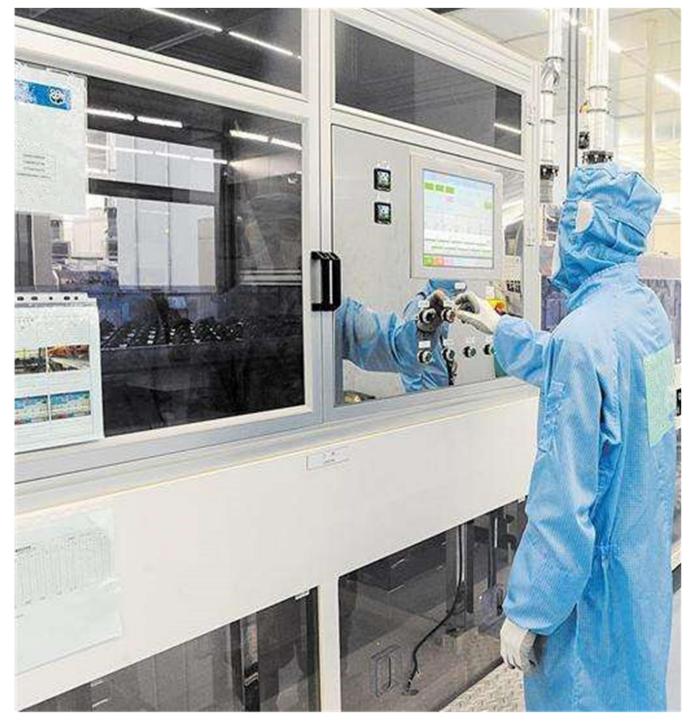

Fig. 6 Cooling monitoring host

\subsection{DSI coefficient}

DSi coefficient has a positive effect on the heat loss of electric vehicle battery, that is, the higher the value level of the former, the greater the total consumption value of the latter, and vice versa. The following table reflects the specific changes of DSI coefficient in the experimental group and the control group within the experimental time of 50min. 
Table 2 Changes of DSI coefficient in experimental group

\begin{tabular}{ccc}
\hline $\begin{array}{c}\text { Testing time } \\
/(\min )\end{array}$ & $\begin{array}{c}\text { DSI } \\
\text { coefficient }\end{array}$ & The average \\
\hline 5 & 0.48 & \\
10 & 0.46 & \\
15 & 0.43 & \\
20 & 0.40 & \\
25 & 0.37 & \\
30 & 0.35 & \\
35 & 0.32 & \\
40 & 0.31 & \\
45 & 0.31 & \\
50 & 0.31 & \\
\hline
\end{tabular}

According to table 2, during the first 35 minutes of the experiment, the DSI coefficient of the experimental group kept a declining trend. After reaching the minimum value of 0.31 , it appeared a stable state for 10 minutes, and the global maximum value was only 0.48 .

\begin{tabular}{ccc}
\multicolumn{3}{c}{ Table 3 Changes of DSI coefficient in control group } \\
\hline $\begin{array}{c}\text { Testing time } \\
\text { / }(\min )\end{array}$ & $\begin{array}{c}\text { DSI } \\
\text { coefficient }\end{array}$ & The average \\
\hline 5 & 0.63 & \\
10 & 0.63 & \\
15 & 0.63 & \\
20 & 0.63 & \\
25 & 0.65 & \\
30 & 0.65 & \\
35 & 0.69 & \\
40 & 0.69 & \\
45 & 0.74 & \\
50 & 0.74
\end{tabular}

According to table 3, DSI coefficient of the control group remained stable in the first 20 minutes of the experiment. From the 25 th minute, there was a step-by-step rising trend. The global maximum value reached 0.74 , far higher than the maximum value of 0.48 in the experiment group.

\subsection{PSI coefficient}

The PSI coefficient also has a positive effect on the heat loss of electric vehicle battery, 
that is, the higher the value level of the former, the greater the total consumption value of the latter, and vice versa. The figure below shows the specific changes of PSI coefficient in the experimental group and the control group within the experimental time of 50min.

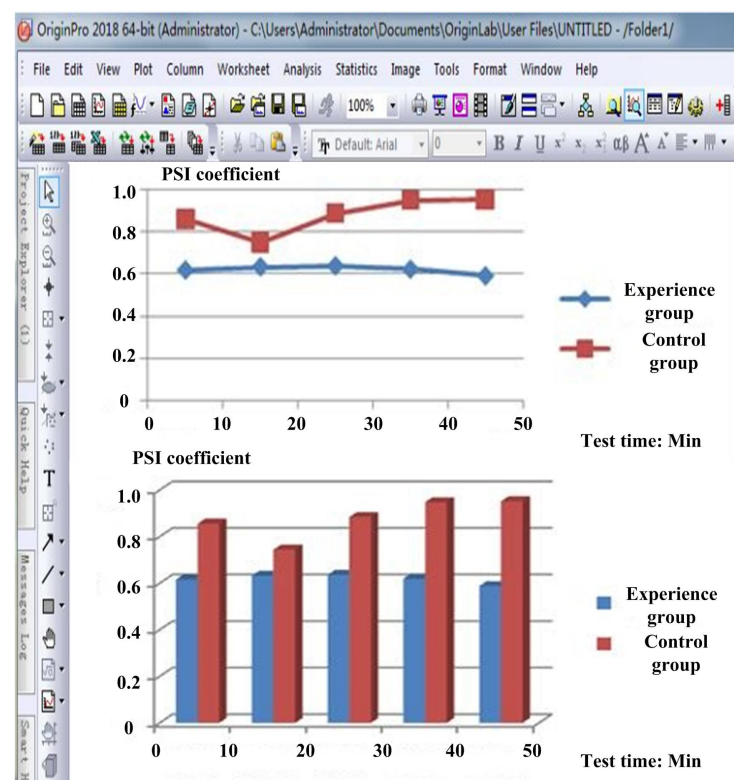

Fig. 7 Comparison of PSI coefficient

It can be seen from Figure 7 that with the increase of experimental time, the PSI coefficient in the experimental group basically keeps a relatively stable trend of change, with the global maximum value only reaching 0.61 ; the PSI coefficient in the control group keeps a state of change of decreasing first and then rising, with the global maximum value reaching 0.98 , far higher than the value level of the experimental group.

\section{Concluding remarks}

Due to the high psi coefficient of the traditional numerical simulation analysis method of the heat dissipation characteristics of the battery box of electric vehicle, the heat dissipation loss of the battery in the process of the electric vehicle moving is high. Therefore, the numerical simulation analysis method of the heat dissipation characteristics of the battery box of electric vehicle based on the coupling model is proposed. Under the support of the coupling model, the numerical simulation analysis method of the thermal characteristics of the battery box of the electric vehicle deals with the comprehensive simulation behavior of the thermal characteristics according to the calculation results of the central difference value, and then obtains the accurate value of the magnetic domain angle deflection. From the practical point of view, DSI coefficient and PSI coefficient show a significant downward trend, which plays a 
very strong control role in reducing the heat loss of battery in the process of electric vehicle moving. In order to further enhance the heat dissipation characteristics of the electric vehicle battery box, the next research will focus on the heat dissipation characteristics of the electric vehicle battery box under different arrangements.

\section{Fund projects}

Research and Innovation Fund of Science and Technology Development Center of Ministry of Education-"Beichuang Assistant" Fund Project : 《Research on Application of New Energy Vehicle Training Platform Intelligent Network Technology》 (number: 2018A05024)

\section{Reference}

[1] Yunlin, S. Rongrong, C. Siming, C.: Study on numerical simulation and optimization of output characteristics of bifacial PV module based on outdoor empirical tests. Renewable Energy Resources. Vol. 24, pp. 1133-1138 (2019)

[2] Yulin, C. Sen, W. Jianwei, Z.: Numerical simulation on dynamic mechanical properties of mixed rock strata based on split hopkinson pressure bar test. Journal of Yangtze River Scientific Research Institute. Vol. 36, pp. 106-111 (2019)

[3] Zhiyong, D, Fengchong, L. Jiqing, C. et al.: Multi-factor coupled heat transfer model with multi-layer and dynamic heat transfer characteristics in vehicle cabin. Journal of Mechanical Engineering. Vol. 55, pp. 145-155 (2019)

[4] Jianpeng, W. Biao, M.: Temperature rise of wet friction pair during sliding in the accelerated life test. Journal of Harbin Institute of Technology. Vol. 51, pp, 103-111 (2019)

[5] Xiaoming, H. Chunyu, S. Jiawei, S. et al.: Analysis on natural convection heat transfer characteristics and multi-objective optimization of structure-parameters for finned heat pipe radiator. Journal of Thermal Science and Technology. Vol. 17, pp. 359-365 (2018)

[6] Ying, C. Ting, L. Qi, J. et al.: Numerical simulation on cavitation flow field characteristics of squeeze film damper based on two-phase flow model. Journal of Aerospace Power. Vol. 34, pp. 1781-1787 (2019)

[7] Zhenkang, Z. Fanyun, Z. Zhiqi, Wa. et al.: Numerical simulation and range analysis of off-design performance for a radial-inflow turbine. The Chinese Journal of Process Engineering. Vol. 19, pp. 246-253 (2019)

[8] Zhongrong, Z. Xinzhe, L. Shu, C.: Numerical simulation of ventilation characteristics in deep-buried long diversion tunnel. Journal of Harbin Engineering University. Vol. 40, pp. 1304-1310 (2019)

[9] Siliang, D. Zhengfei, T. Wenjing, Z. et al.: Numerical simulation and test on aerodynamic characteristics of tandem fan wing. Journal of Beijing University of Aeronautics and Astronautics. Vol. 44, pp. 1164-1175 (2018)

[10] Heyu, F. Chunzhong, Y. Huimin, Ma. et al.: Numerical simulation of fluid-structure interaction 
characteristics between wave and Bohai fixed offshore sea ice. Advances in Marine Science. Vol. 36, pp. 44-54 (2018) 